\title{
Chances and prevention of antibiotic resistance in primary health care: literature review
}

\author{
Omar B. Banamah ${ }^{1 *}$, Mohammed F. Alsamih², Hatem H. Alshehri², Ahmed A. Almutairi², \\ Saud M. Alowayfi², Faisal M. Alshehri' ${ }^{2}$, Mazen F. Alotaibi², Saud H. Shuraym², \\ Abdulaziz M. Alnojeam², Meshari S. Alwadei², Mohammad A. Almohanna², Duaa A. Simsim ${ }^{3}$
}

${ }^{1}$ Consultant of Family Medicine, King Abdulaziz Medical City, National Guarg Health Affairs, Jeddah, Saudi Arabia

${ }^{2}$ College of Medicine, Imam Muhammad Ibn Saud Islamic University, Riyadh, Saudi Arabia

${ }^{3}$ United Pharmaceutical Company, Mecca, Saudi Arabia

Received: 19 December 2020

Revised: 30 December 2020

Accepted: 04 January 2021

*Correspondence:

Dr. Omar B. Banamah,

E-mail: Dr_omarbb@hotmail.com

Copyright: (c) the author(s), publisher and licensee Medip Academy. This is an open-access article distributed under the terms of the Creative Commons Attribution Non-Commercial License, which permits unrestricted non-commercial use, distribution, and reproduction in any medium, provided the original work is properly cited.

\begin{abstract}
Many concerns have been expressed in the field of clinical practice regarding antimicrobial resistance since many infections with severe complications and bad prognosis have been described since the end of the last century. In primary healthcare facilities, the risks for developing high rates of antibiotic resistance is high, probably due to the high prevalence of bacterial infections in these places, and due to the overuse of antibiotics without any relevance of evidence of bacterial infections. For instance, people usually administer high doses of broad-spectrum antibiotics for non-severe, self-limiting respiratory tract infections. Moreover, for fighting bacterial infections, patients usually discontinue the treatment course once the symptoms of the disease were relieved. These factors among many others had led to the high prevalence of antibiotic resistance which may lead to serious complications. Many approaches are being made to reduce the resistance that has been reviewed in this review. These include optimizing the dosage and timing of antibiotics administration which can be achieved by more understanding of the pharmacology of the antibiotics. Moreover, training and education programs that target the prescribers and patients have been proven to be efficient in reducing the rates. These efforts are encouraged to last and target more people for more favorable outcomes.
\end{abstract}

Keywords: Antibiotic, Resistance, Primary care, Education, Prevalence, Prevention

\section{INTRODUCTION}

Many concerns have been expressed in the field of clinical practice regarding antimicrobial resistance since many infections with severe complications and bad prognosis have been described since the end of the last century. Moreover, the prevalence of the resistance has been shockingly increasing in the last few decades which may also contribute to a bad prognosis of the bacterial infection paving the way for more severe reactions that can subsequently lead to severe complications and may even end up in death. ${ }^{1}$ Many organisms have been associated with the high prevalence rates of antimicrobial resistance. Vancomycin-resistant Enterococcus (VRE) species, methicillin-resistant Staphylococcus aureus (MRSA) species, imipenem-resistant Acinetobacter baumannii bacteria, multidrug-resistant (MDR) Pseudomonas aeruginosa, cephalosporin-resistant Escherichia coli, and Klebsiella pneumonia have been regularly reported for this purpose and are said to have a great impact on the prognosis of infected patients. ${ }^{2-4}$

Extended-spectrum $\quad \beta$-lactamase $\quad$ (ESBL)-producing organisms such as Klebsiella pneumonia and Escherichia 
coli and MRSA have been reported as endemic organisms in many hospitals all over the globe. ${ }^{5}$ These types of infections have been also recently reported to occur in local community healthcare facilities. ${ }^{6}$ Among the major resistance problems, fluoroquinolones carbapenem are still sensitive and are considered the last defensive modalities against the different types of Enterobacteriaceae, especially A. baumannii, and $P$. aeruginosa. Therefore, further resistance against such antibiotics may complicate things and induce catastrophes.,7-9 Additionally, the problem of bacterial resistance is not only found with humans, unfortunately, but previous investigations also showed that resistant bacterial isolates of $E$. coli and Salmonella enterica were found in food animals. ${ }^{10,11}$ Resistance to Mycobacterium tuberculosis has been also reported as a concerning problem that threatens public health and especially patients affected by the pathogen. ${ }^{12}$ Besides, Helicobacter pylori infection has been also identified as a major health problem in recent decades due to the rising prevalence rates of several antibiotics which compelled the use of triple and quadruple antibiotic regimens. ${ }^{13-15}$

Primary healthcare facilities are used to describe the small local clinical establishments that usually offer healthcare services for a local, defined population with the quality of service less than that provided by secondary and tertiary hospitals which are considered high-level facilities. Patients at such facilities are more prone to caught infections due to the low levels of sanitation and personal protective measures in addition to the overuse of antibiotics which is attributable to the low level of education and the absence of strict measures by the healthcare officials. Therefore, such places are more prone to high prevalence rates of antibiotic resistance, which we aim to review in this literature and overview of the possible preventive measures.

\section{METHODS}

We performed an extensive literature search of the Medline, Cochrane, and EMBASE databases on 08 November 2020 using the medical subject headings (MeSH) or a combination of all possible related terms. Papers discussing the chances and prevention of antiobiotic resistance in primary health care were screened for relevant information. We did not pose any limits on date, language, and age of participants or publication type.

\section{DISCUSSION}

\section{Chances of antibiotic resistance (prevalence and associated factors)}

The prevalence of antimicrobial resistance is hugely variable among the different types of antibiotics, the targeted bacteria, and the pattern of antibiotic consumption in the reported populations. Wojkowska-Mach et al conducted a ten-year analysis study to study the association between anti-microbial consumption and the prevalence of antibiotic resistance in Poland. ${ }^{16}$ The authors reported that antibiotic consumption in Poland accounted for the highest rate among other European countries included in the analysis. This subsequently leads to an increase in the prevalence of antibiotic resistance in the country from 2007 to 2016. In 2018, Naylor et al conducted a meta-analysis of 214 studies that investigated the global burden of antimicrobial resistance among the included patients and countries. ${ }^{17}$ The authors reported that among the included studies, $48 \%$ of them estimated that antimicrobial resistance was significantly associated with increased mortality rates and healthcare burdens. A systematic review conducted by Shiadeh et al aimed to estimate the global prevalence of antibacterial resistance in certain bacterial isolates including the blood-isolated Enterococcus faecalis and Enterococcus faecium where the authors managed to include 291 studies in the metaanalysis. ${ }^{18}$ The authors estimated that the prevalence rate was variable by country and according to the culture. Enterococcus faecalis was found be highly resistant to quinupristin/dalfopristin $(97 \%)$ while the lowest resistance rate was found to be associated with linezolid antibiotics $(0.8 \%)$. Moreover, the authors reported that America, Europe, and countires of the western pacific accounted for the lowest prevalence rate among other countries for linezolid and vancomycin antibiotics. Resistance to vancomycin was also found to be highest in South-East Asia and Eastern Mediterranean regions while analysis by country showed that Sweden possessed the lowest prevalence rate while Japan and Nepal recorded the highest. Enterococcus faecium showed the highest resistance of penicillin $(85 \%)$ while resistance to tetracycline $(1 \%)$ and linezolid $(1.7 \%)$ was the lowest. The reported antibiotic resistance by country showed that the Netherlands had the lowest prevalence rates $(0.1)$ while Brazil had the highest $(67 \%)$ for this organism. Based on these results, the prevalence of antimicrobial resistance seems to increase by time as authors reported that, for instance, resistance to vancomycin in the aforementioned organisms increased by time from 2000 to 2016. Vancomycin-resistant enterococci (VRE) prevalence rate was also variable among studies in the literature. In Ethiopia, Melese et al. estimated the prevalence rate to be $14.8 \%$ (95\% CI: 8.7-24.3) while in Nigeria, Wada et al estimated the prevalence of VRE from 19 original investigations to be $25.3 \% \quad(95 \% \quad \mathrm{CI}: \quad 19.8-30.8 \%)$ Acinetobacter baumannii resistance to antibiotics was also reported by many previous investigations which showed that the prevalence rate of this resistance was hugely variable according to the applied antibiotic per country. ${ }^{19,20}$ Pormohammad et al analyzed the results of 150 original investigations in a single meta-analysis to estimate the prevalence rate of cefotaxime and colistin by country. ${ }^{21}$ Africa had a $99 \%$ prevalence resistance rate to cefotaxime which was the highest while countries in the Western Pacific had the lowest prevalence rate of $1.1 \%$. Moreover, the analysis showed that Germany $(0.2 \%)$ had the lowest prevalence rate for colistin while Lebanon (17.5\%), and China (12\%) possessed the highest rate for the same antibiotic. Another meta-analysis by Pormohammad et al 
showed that $E$. Coli was highly resistant to amoxicillin (70.5\%; 9\% CI: $57.5 \%-81 \%$ ) while resistance to colistin was found to be the lowest for this organism $(0.8 \%$; $95 \%$ CI $0.2 \%-3.8 \%$ ) in human isolates. ${ }^{22}$ Other associated factors are related to the patient's demographics, health status, and the presence of other comorbidities that may lower the patient's immunity. ${ }^{23}$ Moreover, factors related to the type and efficacy of the healthcare facilities and experts have been also reported. ${ }^{24}$ These results indicate the fact that antibiotic resistance is highly variable per each orgnism and for each antibiotic which is hard to cover in a single report. However, we hope that we shed more light on the seriousness of the issue and that serious preventive measures should be considered to reduce these rates and enhance the prognosis of eliminating such orgnisms and reducing their mobidities.

\section{Preventive measures for reducing the resistance}

Although antibiotic resistance has been a major problem in the past years with many mortalities and complications and no effective management modalities, previously published studies proposed various approaches to reduce the prevalence of the resistance. These measures are discussed as follows from the findings of previous investigations.

\section{Optimizing the antibiotic}

The antimicrobial stewardship programs (ASPs) have been now widely accepted by many institutions worldwide in this field. ${ }^{25}$ It has been first proposed by the infection diseases society of America (IDSA) and the society of healthcare epidemiology of America (SHEA) in 2007. ${ }^{26}$ These programs are built on previous investigations on different types of antibiotic resistance materials and their organisms. These include vancomycin and VRE, fluoroquinolones, and MRSA, cephalosporine, and cephalosporine-resistant Enterobacteriaceae, in addition to carbapenem in pseudomonas, carbapenem-resistant Acinetobacter, and Enterobacteriaceae. ${ }^{27-33}$ The program targets education about antibacterial resistance in addition to the front and back-end strategies that are targeted to limit the availability of certain antibiotics to the public and reconsidering the use of broad-spectrum antibiotics in addition to applying the most relevant antibiotics based on the susceptibility test results. ${ }^{34,35}$ Many aspects have been also reported in such programs. These include: adapting the proper antibiotic in a situation-specific pattern; providing better education about the strategy for healthcare providers; results of antimicrobial testing; identification and culturing of the right organisms; dose optimization and proper treatment courses based on the more comprehension of the antibiotic pharmacodynamics and kinetics, in addition to; and preauthorization of absolute antibiotic intake. The latter aspect has been named as the main strategy according to the developing organizations and according to previous reports which showed that it is the main effective modality that can lead to a great reduction in the resistance rate by reducing the frequency of their intake..$^{35-38}$ The relationship between antibiotic underdosing and discontinuation of the course with the development of the resistance was first proposed by Stamey et al. ${ }^{39}$ Drew et al indicated that the application of this program's criteria will lead to a much reduction in the resistance rates. ${ }^{28}$ Cook et al showed that $P$. aeruginosa susceptibility to carbapenem improves by $10-25 \%$ by restricting the use of ciprofloxacin by $90 \%$ between 2009 and $2010 .^{27}$

Dose optimization is also an important factor as many healthcare providers usually prescribe unnecessary antibiotics for their patients. For instance, antibiotics are widely prescribed in idiopathic respiratory tract infections although they are self-limiting are usually treated by symptomatic approaches as previous studies showed that the benefit from prescribing antibiotics for respiratory tract infections is usually minimal and can be only used in severe complications contributing to secondary infection on top of the lesion. ${ }^{40-42}$ In the same context, Hay et al showed concerns about why $88 \%$ of patients with selflimiting respiratory tract infections are treated with antibiotics. ${ }^{43}$ This phenomenon differs greatly by country and region as previous investigations showed that it is associated with culture, socio-economic level, clinical autonomy, country background, and the cultural beliefs of the patients and prescribers. ${ }^{44-46}$ Hulscher et al expressed that in such countries, people are directed in this path by non-scientific misleading ideas. ${ }^{46}$ Besides, antibiotics prescription is mainly based on personal suggestions of the prescribers not on scientific-based manners that require the identification of the infective organisms. ${ }^{44-46}$ In a review by Harbart et al the authors showed indicated this fact where antibiotics are being prescribed in association with diagnostic uncertainty. ${ }^{47}$ The authors also showed that Southern European countries are more prone to antibiotic resistance than egalitarian societies due to the high intake and prescription of antibiotics in these countries. The socio-economic level of some countries has been also proven to take part in the over usage of antibiotics which results from the pressure of many pharmaceutical companies. Kirby et al showed that antimicrobial resistance differed from patients according to their economic incomes. ${ }^{48}$ Another important factor that may influence antibiotic prescription is the attitude of the prescribing agents. Cole et al conducted a survey on 1000 general practitioners and found that $55 \%$ of them were influenced by patients' pressure to prescribe antibiotics while $44 \%$ prescribed antibiotics for patients to motivate them for leaving the surgical platform. ${ }^{49}$ These factors indicate that optimization of antibiotic use is essential in decreasing the resistance, and therefore, effective measures should be approached targeting the patients, clinicians and on a local and global level.

\section{Education about antibiotic resistance}

In general, educational events can reduce the inappropriate behavior about drug prescription as indicated by Kunstler et al which summarized the results of 14 reviews on this topic. ${ }^{50}$ Education about antibiotic resistance and about the 
complications that may result from the phenomenon might be effective in reducing unnecessary consumption of antibiotics, and consequently, reduce the resistance. Education is directed to many audiences including prescribers, pharmacists, healthcare officials, and patients. The success of the ASPs has been also mostly due to successful training and education of GPs towards the daily prescriptions and reducing antibiotic consumption unless needed. ${ }^{51}$ Rodrigues et al conducted a clinical trial to investigate the efficacy of applying education as an interventional approach for patients, physicians, and pharmacists and its efficacy on improving antibiotic resistance to many organisms in primary care. ${ }^{52}$ The authors concluded that significant improvements were noticed regarding the prescription of penicillin combinations including $\beta$-lactamase inhibitors, third- and fourth-generation cephalosporins, and fluoroquinolones $\beta$ lactamase-sensitive penicillins, and the ratio of broad- to narrow-spectrum antibiotics. The frequency of educational outreach (EO) might also influence the results of antibiotic prescription. Chhina et al reported that after a single EO to their GPs by a therapeutic advisor, a significant increase in the non-recommended prescription of antibiotics was reduced by $74 \%$ in the EO group. ${ }^{53}$ Clyne et al also showed that applying educational interventions significantly reduced the un-needed antibiotic prescription by the included participants. ${ }^{54}$ Additionally, a meta-analysis of randomized controlled trials conducted by Fleming et al to assess the effect of educational interventions for physicians and nurses on antibiotic prescription in long-term healthcare facilities. ${ }^{54}$ The authors estimated that, although the evidence was low in this study due to heterogeneity, educational approaches might influence the inappropriate prescribtion patterns of antibiotics in these facilities. In addition to educting physicians and pharmacists, previous studies also showed that other educational programs targeted patients and children to spread awareness about the benefits and dangers of antibiotics resistance. ${ }^{55}$ Such progrmas may have an important role in influencing the public's understanding about the unnecessary and overuse of antibiotics, and therefore, may reduce the resistance rates. ${ }^{56}$

\section{Other interventional procedures}

Hygiene and conducting disinfection in primary healthcare facilities have been also reported by previous investigations as preventive approaches against antibiotic resistance. It has been estimated that around 1.7 million cases of hospital-acquired infections occur each year which results in the development of severe complications and deaths in thousands of them. ${ }^{57}$ Therefore, sticking to personal protective measures whenever in the hospital is highly recommended to avoid getting infected. ${ }^{58}$ Personal protective measures might include continuous use of hand sanitizers, frequently washing hands, and wearing masks. ${ }^{59}$ This can effectively reduce the incidence of infection and consequently reduce the need to administrate large amounts of antibiotics which can intervene against bacterial resistance. Lee et al also reviewed some other procedures to decrease the burden of antibiotic resistance. ${ }^{59}$ They reported that using antibiotics in veterinary medicine might have also contributed to the overall emergence of resistance in humans as many of these approaches were not for therapeutic purposes in the first place, and therefore, restricting the use of these antibiotics should be done. Moreover, the study reviewed the previous literature which indicates the emerging novel ideas of probiotics and novel antibiotics which may replace the existing non-bacterial sensitive ones.

\section{CONCLUSION}

Antibiotic resistance will remain an issue of concern in the years to follow due to the uprising prevalence rates of this phenomenon. Public health policies are urged to take action to reduce consumptions and spread awareness about the hazards of this situation which may affect many lives. Many approaches are being made to reduce the resistance that has been reviewed in this review. These include optimizing the dosage and timing of antibiotics administration which can be achieved by more understanding of the pharmacology of the antibiotics. Moreover, training and education programs that target the prescribers and patients have been proven to be efficient in reducing the rates. These efforts are encouraged to last and target more people for more favourable outcomes.

Funding: No funding sources Conflict of interest: None declared Ethical approval: Not required

\section{REFERENCES}

1. Palmer AC, Kishony R. Understanding, predicting and manipulating the genotypic evolution of antibiotic resistance. Nature Rev Genetics. 2013;14(4):243-8.

2. Meyer E, Schwab F, Schroeren-Boersch B, Gastmeier P. Dramatic increase of third-generation cephalosporin-resistant E. coli in German intensive care units: secular trends in antibiotic drug use and bacterial resistance, 2001 to 2008. Crit Care. 2010;14(3):113.

3. Rossolini GM, Mantengoli E, Docquier J, Musmanno RA, Coratza G. Epidemiology of infections caused by multiresistant gram-negatives: ESBLs, MBLs, panresistant strains. Microbiologica-bologna. 2007;30(3):332.

4. Spellberg B, Guidos R, Gilbert D. The epidemic of antibiotic-resistant infections: a call to action for the medical community from the Infectious Diseases Society of America. Clin Infect Dis. 2008;46(2):15564.

5. Cantas L, Shah SQA, Cavaco LM. A brief multidisciplinary review on antimicrobial resistance in medicine and its linkage to the global environmental microbiota. Front Microbiol. 2013;4:96.

6. Otter JA, French GL. Molecular epidemiology of community-associated methicillin-resistant 
Staphylococcus aureus in Europe. Lancet Infect Dis. 2010;10(4):227-39.

7. Brown S, Bantar C. Limitation of Acinetobacter baumannii treatment by plasmid-mediated carbapenemase ARI-2. Lancet. 1998;351(9097):1867.

8. Poirel L, Nordmann P. Carbapenem resistance in Acinetobacter baumannii: mechanisms and epidemiology. Clin Microbiol Infecti. 2006;12(9):826-836.

9. Strahilevitz J, Jacoby GA, Hooper DC, Robicsek A. Plasmid-mediated quinolone resistance: a multifaceted threat. Clinical microbiology reviews. 2009;22(4):664-89.

10. Fischer J, Rodríguez I, Schmoger S. Salmonella enterica subsp. enterica producing VIM-1 carbapenemase isolated from livestock farms. J Antimicrob Chemother. 2013;68(2):478-80.

11. Fischer J, Rodríguez I, Schmoger S. Escherichia coli producing VIM-1 carbapenemase isolated on a pig farm. J Antimicrob Chemother. 2012;67(7):1793-5.

12. Almeida Da Silva PE, Palomino JC. Molecular basis and mechanisms of drug resistance in Mycobacterium tuberculosis: classical and new drugs. J Antimicrob Chemother. 2011;66(7):141730 .

13. Thieu HV, Bach Dat B, Nam NH. Antibiotic resistance of Helicobacter pylori infection in a children's hospital in Vietnam: prevalence and associated factors. Minerva Med. 2020.

14. Khoder G, Muhammad JS, Mahmoud I, Soliman SSM, Burucoa C. Prevalence of Helicobacter pylori and Its Associated Factors among Healthy Asymptomatic Residents in the United Arab Emirates. Pathogens. 2019;8(2):44.

15. Harris A. Treatment of Helicobacter pylori. World J Gastroenterol. 2001;7(3):303-7.

16. Wojkowska-Mach J, Godman B, Glassman A. Antibiotic consumption and antimicrobial resistance in Poland; findings and implications. Antimicrob Resist Infect Control. 2018;7:136-7.

17. Naylor NR, Atun R, Zhu N. Estimating the burden of antimicrobial resistance: a systematic literature review. Antimicrob Resist Infect Control. 2018;7(1):58.

18. Jabbari Shiadeh SM, Pormohammad A, Hashemi A, Lak P. Global prevalence of antibiotic resistance in blood-isolated Enterococcus faecalis and Enterococcus faecium: a systematic review and metaanalysis. Infect Drug Resist. 2019;12:2713-25.

19. Melese A, Genet C, Andualem T. Prevalence of Vancomycin resistant enterococci (VRE) in Ethiopia: a systematic review and meta-analysis. BMC Infect Dis. 2020;20(1):124.

20. Wada Y, Harun AB, Yean CY, Zaidah AR. Vancomycin-Resistant Enterococci (VRE) in Nigeria: The First Systematic Review and MetaAnalysis. Antibiotics (Basel). 2020;9(9):565.

21. Pormohammad A, Mehdinejadiani K, Gholizadeh P. Global prevalence of colistin resistance in clinical isolates of Acinetobacter baumannii: A systematic review and meta-analysis. Microbial Pathogenesis. 2020;139:103887.

22. Pormohammad A, Nasiri MJ, Azimi T. Prevalence of antibiotic resistance in Escherichia coli strains simultaneously isolated from humans, animals, food, and the environment: a systematic review and metaanalysis. Infect Drug Resist. 2019;12:1181-97.

23. Wolfe CM, Cohen B, Larson E. Prevalence and risk factors for antibiotic-resistant community-associated bloodstream infections. Journal of Infection and Public Health. 2014;7(3):224-232.

24. Iroh Tam P-Y, Koopmeiners JS. Trends in Pneumonia Hospitalizations in Hennepin County, Minnesota, 1999-2010. Mayo Clinic Proceedings. 2013;88(10):1181-2.

25. Owens RC. Antimicrobial stewardship: concepts and strategies in the 21st century. Diagnost Microbiol Infect Dis. 2008;61(1):110-28.

26. Dellit TH, Owens RC, McGowan JE. Infectious Diseases Society of America and the Society for Healthcare Epidemiology of America guidelines for developing an institutional program to enhance antimicrobial stewardship. Clin Infect Dis. 2007;44(2):159-77.

27. Cook PP, Rizzo S, Gooch M, Jordan M, Fang X, Hudson S. Sustained reduction in antimicrobial use and decrease in methicillin-resistant Staphylococcus aureus and Clostridium difficile infections following implementation of an electronic medical record at a tertiary-care teaching hospital. J Antimicrob Chemother. 2011;66(1):205-9.

28. Drew RH. Antimicrobial stewardship programs: how to start and steer a successful program. J Managed Care Pharm. 2009;15(2):18-23.

29. Madaras-Kelly KJ, Remington RE, Lewis PG, Stevens DL. Evaluation of an intervention designed to decrease the rate of nosocomial methicillinresistant Staphylococcus aureus infection by encouraging decreased fluoroquinolone use. Infect Control Hospital Epidemiol. 2006;27(2):155-69.

30. Harbarth S, Cosgrove S, Carmeli Y. Effects of antibiotics on nosocomial epidemiology of vancomycin-resistant enterococci. Antimicrob Agents Chemother. 2002;46(6):1619-28.

31. Calil R, Marba STM, von Nowakonski A, Tresoldi AT. Reduction in colonization and nosocomial infection by multiresistant bacteria in a neonatal unit after institution of educational measures and restriction in the use of cephalosporins. Am J Infect Control. 2001;29(3):133-8.

32. Go ES, Urban C, Burns J. Clinical and molecular epidemiology of Acinetobacter infections sensitive only to polymyxin B and sulbactam. Lancet. 1994;344(8933):1329-32.

33. Rahal JJ, Urban C, Segal-Maurer S. Nosocomial antibiotic resistance in multiple gram-negative species: experience at one hospital with squeezing the resistance balloon at multiple sites. Clin Infect Dis. 2002;34(4):499-503. 
34. Moehring RW, Anderson DJ. Antimicrobial stewardship as part of the infection prevention effort. Curr Infect Dis Rep. 2012;14(6):592-600.

35. Paterson DL. The role of antimicrobial management programs in optimizing antibiotic prescribing within hospitals. Clinical infectious diseases. 2006;42(2):90-5.

36. Beardsley JR, Williamson JC, Johnson JW, Luther VP, Wrenn RH, Ohl CC. Show me the money: longterm financial impact of an antimicrobial stewardship program. Infect Control Hospital Epidemiol. 2012;33(4):398-400.

37. Carling P, Fung T, Killion A, Terrin N, Barza M. Favorable impact of a multidisciplinary antibiotic management program conducted during 7 years. Infect Control Hospital Epidemiol. 2003;24(9):699706.

38. Standiford HC, Chan S, Tripoli M, Weekes E, Forrest GN. Antimicrobial stewardship at a large tertiary care academic medical center: cost analysis before, during, and after a 7-year program. Infect Control Hospital Epidemiol. 2012;33(4):338-45.

39. Stamey TA. Resistance to nalidixic acid: a misconception due to underdosage. JAMA. 1976;236(16):1857-60.

40. Ashworth M, Charlton J, Ballard K, Latinovic R, Gulliford M. Variations in antibiotic prescribing and consultation rates for acute respiratory infection in UK general practices 1995-2000. Br J Gen Pract. 2005;55(517):603-8.

41. Smith SM, Fahey T, Smucny J, Becker LA. Antibiotics for acute bronchitis. Cochrane Database Systematic Rev. 2014(3).

42. Venekamp RP, Sanders S, Glasziou PP, Del Mar CB, Rovers MM. Antibiotics for acute otitis media in children. Cochrane Database of Systematic Rev. 2013(1).

43. Hay AD, Tilling K. Can $88 \%$ of patients with acute lower respiratory infection all be special? Br J Gen Pract. 2014;64(619):60-2.

44. Butler CC, Rollnick S, Pill R, Maggs-Rapport F, Stott N. Understanding the culture of prescribing: qualitative study of general practitioners' and patients' perceptions of antibiotics for sore throats. BMJ. 1998;317(7159):637-42.

45. Moore M, McNulty C. European Antibiotic Awareness Day 2012: TARGET antibiotics through guidance, education, and tools. $\mathrm{Br} \mathrm{J}$ Gen Pract. 2012;62(605):621-2.

46. Hulscher MEJL, Grol RPTM, van der Meer JWM. Antibiotic prescribing in hospitals: a social and behavioural scientific approach. Lancet Infect Dis. 2010;10(3):167-75.

47. Harbarth S, Samore MH. Antimicrobial resistance determinants and future control. Emerg Infect Dis. 2005;11(6):794-801.

48. Kirby A, Herbert A. Correlations between Income inequality and antimicrobial resistance. PLoS One. 2013;8(8):73115-6.
49. Cole A. GPs feel pressurised to prescribe unnecessary antibiotics, survey finds. BMJ : $\mathrm{Br}$ Med $\mathrm{J}$. 2014;349:5238.

50. Kunstler BE, Lennox A, Bragge P. Changing prescribing behaviours with educational outreach: an overview of evidence and practice. BMC Med Educ. 2019;19(1):311-2.

51. Cantón R, Bryan J. Global antimicrobial resistance: from surveillance to stewardship. Part 2: stewardship initiatives. Expert Rev Anti-infective Ther. 2012;10(12):1375-7.

52. Teixeira Rodrigues A, Roque F, Piñeiro-Lamas M, Falcão A, Figueiras A, Herdeiro MT. Effectiveness of an intervention to improve antibiotic-prescribing behaviour in primary care: a controlled, interrupted time-series study. J Antimicrob Chemother. 2019;74(9):2788-96.

53. Chhina H, Bhole V, Goldsmith C, Hall W, Kaczorowski J, Lacaille D. Effectiveness of Academic Detailing to Optimize Medication Prescribing Behaviour of Family Physicians. J Pharm Pharmaceutic Sci. 2013;16:511-29.

54. Fleming A, Browne J, Byrne S. The Effect of Interventions to Reduce Potentially Inappropriate Antibiotic Prescribing in Long-Term Care Facilities: a Systematic Review of Randomised Controlled Trials. Drugs Aging. 2013;30(6):401-8.

55. McNulty CAM, Lecky DM, Farrell D, et al. Overview of e-Bug: an antibiotic and hygiene educational resource for schools. J Antimicrob Chemother. 2011;66(5):3-12.

56. Huttner B, Goossens H, Verheij T, Harbarth S. Characteristics and outcomes of public campaigns aimed at improving the use of antibiotics in outpatients in high-income countries. Lancet Infect Dis. 2010;10(1):17-31.

57. Weber DJ, Rutala WA, Miller MB, Huslage K, Sickbert-Bennett E. Role of hospital surfaces in the transmission of emerging health care-associated pathogens: Norovirus, <em $>$ Clostridium difficile $</$ em $>$, and <em $>$ Acinetobacter $</ e m>$ species. Am J Infect Control. 2010;38(5):25-33.

58. Conly JM, Hill S, Ross J, Lertzman J, Louie TJ. Handwashing practices in an intensive care unit: The effects of an educational program and its relationship to infection rates. Am $\mathbf{J}$ Infect Control. 1989;17(6):330-9.

59. Lee C-R, Cho IH, Jeong BC, Lee SH. Strategies to minimize antibiotic resistance. Int $\mathrm{J}$ Environ Res Public Health. 2013;10(9):4274-305.

Cite this article as: Banamah $\mathrm{OB}$, Alsamih MF, Alshehri HH, Almutairi AA, Alowayfi SM, Alshehri FM, et al. Chances and prevention of antibiotic resistance in primary health care: literature review. Int J Community Med Public Health 2021;8:875-80. 